\title{
A model for the bubble lift-off diameter in subcooled boiling flow in a horizontal channel
}

\author{
S. Shabannejad \& N. Ashgriz \\ Department of Mechanical and Industrial Engineering, \\ University of Toronto, Canada
}

\begin{abstract}
Bubble nucleation in subcooled boiling flow in a horizontal annular channel with a square cross section is investigated. The experiments were performed with water at pressures of between 1 and $2.5 \mathrm{~atm}$ and at low heat and mass fluxes. Bubble lift-off diameters were obtained from direct high speed videography. The developed model for the bubble lift-off diameter was obtained by analyzing the forces acting on a bubble and then a model for the bubble growth rate constant was suggested. Our proposed model was then validated by experimental data and it agreed well with the experimental data. The obtained results were also compared with the available correlations from other researchers.
\end{abstract}

Keywords: sub-cooled boiling, forced convection heat transfer, bubble lift-off diameter, flow visualization with high-speed camera, critical heat flux, horizontal annulus, nucleation.

\section{Introduction}

The flow inside a Pressurized Heavy Water Reactor (PHWR) core is subcooled, which is generally characterized by the formation of bubbles on the heated surface. The characteristics of the bubbles formed depend on the heat flux, mass flux, subcooling, and pressure, among the other flow parameters. Numerous studies have been conducted to determine the bubble nucleation characteristics, such as the bubble diameter, bubble velocity and its frequency.

This paper focuses on the bubble nucleation on a horizontally heated wall under low heat flux, low experimental pressure, and low flow velocity conditions. After the bubble nucleates at its nucleation site, it grows and then departs its site. This is the departure moment and its corresponding bubble 
diameter is called the bubble departure diameter. After the departure, the bubble slides a certain distance on the heater surface while it grows due to the heat transfer from the heater surface underneath it. The bubble lifts off the surface when it reaches a certain size. Its diameter at this moment is referred to as the lift-off diameter [1]. Several studies have been performed to measure the bubble departure and lift-off diameters. Four of the commonly used lift-off diameter models are shown in table 1.

Table 1: Bubble Lift-off diameters for forced convective boiling flows.

\begin{tabular}{|c|c|}
\hline Bubble Lift-off Diameter & Author \\
\hline$D_{l o}=\frac{4 \sqrt{22 / 3} b^{2} v_{f}}{\pi u_{r} \sqrt{C_{s l}}} J a^{2} \operatorname{Pr}_{\mathrm{f}}^{-1}$ & Situ et al. [2] \\
\hline $\begin{array}{l}D_{l o}=\frac{A \rho_{f} \alpha_{f}^{2}}{\sigma} J a^{B}\left(\frac{T_{w}-T_{f}}{\Delta T_{s a t}}\right)^{C}\left(\frac{\rho_{f}}{\rho_{g}}\right)^{D} B o^{E} \\
\mathrm{~A}=440.98, \mathrm{~B}=-0.708, \mathrm{C}=-1.112, \mathrm{D}=1.747, \mathrm{E}=0.124\end{array}$ & $\begin{array}{l}\text { Prodanovic et } \\
\text { al. [3] }\end{array}$ \\
\hline $\begin{array}{l}D_{l o}=1.3\left(\sin \theta_{s}\right)^{0.4} L\left[0.2 e^{-1.28 \times 10^{-4} R e_{f}+}\right. \\
0.005] J a^{0.45} e^{-0.0065 J a_{s u b}} \\
L=\sqrt{\frac{\sigma}{g\left(\rho_{f}-\rho_{g}\right)}}, J a_{s u b}=\frac{\rho_{f} c_{p f} \Delta T_{s u b}}{\rho_{g} h_{f g}}\end{array}$ & Basu et al. [4] \\
\hline $\begin{array}{l}D_{\text {max }}=2.42 \times 10^{-5} P^{0.709} \frac{a}{\sqrt{b \Omega}} \\
a=\frac{\Delta T_{s a t} k_{f} \gamma}{2 \rho_{g} H_{f g}\left(\pi \alpha_{f}\right)^{1 / 2}}, \quad b=\frac{\Delta T_{s u b}}{2\left(1-\frac{\rho_{g}}{\rho_{l}}\right)}, \gamma= \\
\left(\frac{k_{s} \rho_{s} C_{s}}{k_{f} \rho_{f} C_{p, f}}\right)^{1 / 2}, \quad \phi=\max \left(\frac{U_{b u l k}}{0.61}, 1\right)^{0.47}\end{array}$ & Unal [5] \\
\hline
\end{tabular}

Unal [5] obtained a model based on an energy balance for a single bubble in order to predict the maximum bubble diameter. Zeng et al. [6] and Klausner et al. [1] performed experiments in a horizontal rectangular channel using saturated R-113, while Prodanovic et al. [3] and Situ et al. [2] did their experiments in vertical channels using water to find the bubble lift-off diameters. In order to develop mechanistic models for the bubble departure and lift-off diameters, the forces acting on a single bubble were analyzed and force balance equations were solved. This was first done by Klausner [1] and later, Zeng simplified Klausner's model by assuming a negligible lift-off contact angle and shear lift force. They used Zuber's [7] model for bubble growth rates:

$$
r_{b}=\frac{2 b}{\sqrt{\pi}} J a \sqrt{\alpha_{f} t}
$$

Klausner et al. [1] suggested a growth rate constant of $b=17.3$ for flows in horizontal channels. The developed models were then validated against 
experimental data, and were compared to existing correlations for bubble diameter. In this study, Klausner [1] and Zeng [6] methods were used and a model for $b$ as a function of pressure based on experimental data is suggested.

\section{Experimental procedure}

\subsection{Experimental test facility}

The experimental setup had three sub-assemblies: Tank assembly (water source), Test section (horizontal channel), and Visualization system (high-speed camera). The tank assembly included an isolated tank, a pump, a cartridge heater and a temperature control device, i.e. thermocouple. The water was first preheated to the desired temperature and then was pumped into pipelines connected to the test section. The test section was an annulus with a square cross section (ID dimensions: $19.84 \mathrm{~mm} \times 27.68 \mathrm{~mm}$, length of $500 \mathrm{~mm}$ ) made of aluminium with high resistance glass windows on two sides for visualization purposes. The heater rod was of stainless steel and was placed inside a zirconium tube. This was done in order to have the exact same surface conditions and roughness as the cladding in the most prevalent HPWRs. The electrical heater had the ability to generate a heat flux of up to $124 \mathrm{~kW} / \mathrm{m}^{2}$. The K-type thermocouple embedded between the steel heating rod and the $\mathrm{Zr}-4$ shell was used to measure the temperature at the interface of the stainless steel heater and $\mathrm{Zr}-4$ shell to give a first approximation of the wall temperature. The OD of the zirconium tube was $13.095 \mathrm{~mm}$ and thus, the hydraulic diameter of the chamber was calculated as $12.17 \mathrm{~mm}$. Fig. 1 shows a schematic of the experimental setup.

\subsection{Digital photographic method for visualization}

A high-speed CCD video camera was used to record and measure bubble diameter, velocity, and nucleation process. A modified slider mechanism was used to create a 2-DOF-system. This allowed the user to make small changes in the $x$ - and $y$-directions. The camera was then mounted on a 1-D traverse rail that could be moved forward and backward relative to the test section. Lighting was also an important issue in the camera assembly. The camera was adjusted to focus on an active nucleation site and in order to capture the very short bubble growth period, the camera frame rate was set to 4000 frames per second (fps). The high-speed camera had a flash-synchronized shutter speed of 1/8000 s, a total of 2.74 million pixels CCD (record pixels $128 \times 512$ ), and a direct connection to a computer which was used to process images. After capturing the images, in order to correct the contrast and brightness of the images and also to obtain the necessary data regarding the bubbles, image processing was performed using MatLAB. The accuracy of the results was checked using ImageJ software. Fig. 2 shows typical consecutive images of bubble nucleation, departure and lift-off at a pressure of $1.5 \mathrm{~atm}$, mass flux of $300 \mathrm{~kg} / \mathrm{m}^{2} \mathrm{~s}$, heat flux of $124 \mathrm{~kW} / \mathrm{m}^{2}$, and subcooling of $9^{\circ} \mathrm{C}$. 


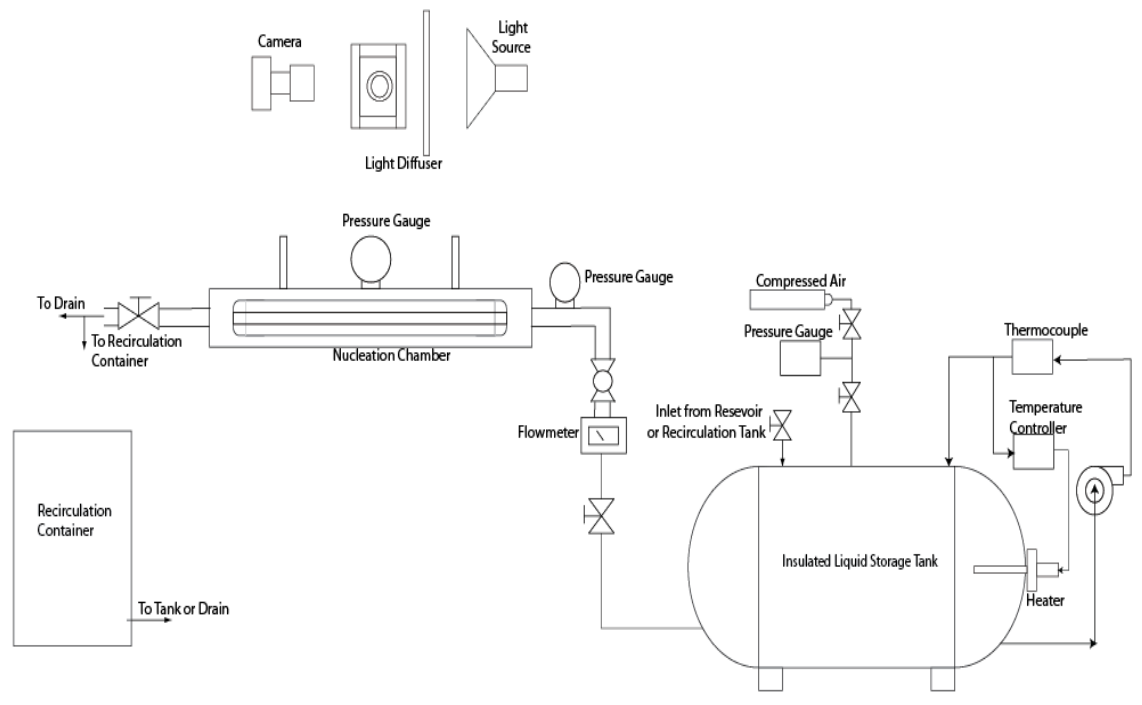

Figure 1: Schematic of the experimental setup.

\subsection{Experimental conditions and errors}

Experiments of 37 cases were conducted for the study of the bubble lift-off diameter. The requirements of the project were as follows: 1-2.5 atm pressure, $32-1{ }^{\circ} \mathrm{C}$ subcooling temperature (i.e. difference between the bulk temperatures of water flow and their corresponding saturated temperatures), low turbulence, $0.3-$ $0.4 \mathrm{~m} / \mathrm{s}$ inlet water velocity, and $124 \mathrm{~kW} / \mathrm{m}^{2}$ heat flux. In order to have more accurate results, distilled water was used and was passed through a purifier and demineralizer for 24 hours before each series of tests in order to remove all impurities. The dissolved air was also removed by boiling the water in the storage tank for three to four hours. Once the test was started and steady state was reached at the test pressure and temperature, the video recording of the bubble nucleation was initiated, which lasted 6 seconds.

Calibration of the flow meter was performed and its accuracy was given as $\pm 0.5 \%$. The temperature of the fluid and the heater surface were measured by four K-type thermocouples: one placed at the inlet to the chamber, two inside the test section, and one underneath the heater surface. The pressure was also measured at the test section by a single scale multi-purpose pressure gauge. The uncertainties associated with the applied heat and mass fluxes were $\pm 1.0 \%$ and $\pm 1.3 \%$, respectively. The uncertainties for the pressure gauges and thermocouples were $\pm 5 \mathrm{kPa}$ and $\pm 0.5^{\circ} \mathrm{C}$, respectively. The error associated with image processing was up to 2 pixels around the circumference of the bubble (i.e. $\sim 30-40 \mu \mathrm{m}$ error). 


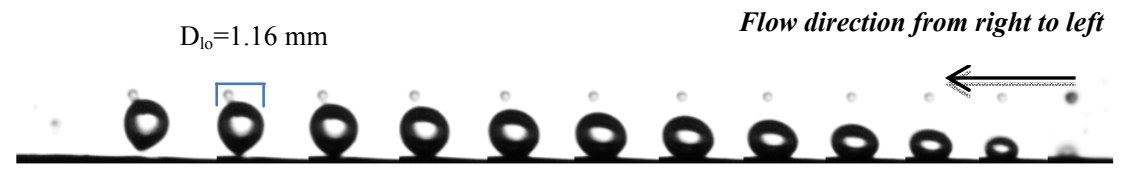

Figure 2: Consecutive images of the nucleation to lift-off process for a bubble. (Image sequence from right to left, starts at $\mathrm{t}=0.25 \mathrm{~ms}$, ends at $\mathrm{t}=3.0 \mathrm{~ms}$, with time step of $0.25 \mathrm{~ms}$.)

\section{Results and discussion}

\subsection{Experimental analysis}

Upon analyzing the recorded images for the bubble behaviours, it was found that there were significant differences in bubble sizes and lifetimes for bubbles under the same experimental conditions. There were two main causes for such variations: (1) existing cavities on the surface had different sizes, which resulted in various bubble sizes, and/or (2) bubbles were experiencing varying local temporal and velocity fields. Prodanovic et al. [3] mentioned the latter effects as dominant in creating scatter in experimental data. Surface roughness also played an important role in initiating the nucleation and bubble diameter. In this study, the average surface roughness was 0.1 , since the maximum roughness was around 0.6 .

Bubble lift-off diameter was measured from all the experimental tests and was averaged over all the nucleation sites. It was found that the experimental conditions covered the late stage of the partial boiling region and the early stage of the fully developed region depending on the flow and heat flux conditions.

The experimentally determined bubble lift diameters were plotted versus mass flux, pressure and subcooling temperature. Fig. 3 shows the effect of liquid pressure on bubble lift-off diameter. The lift-off diameter reduces with increasing fluid pressure at all mass fluxes and subcooling temperatures. The same result was obtained by previous research (e.g. Tolubinsky and Kostanchuk [8]; Prodanovic et al. [3]). However, in contrast to Tolubinsky's work, the present study finds that bubble lifetime and sliding time decrease with an increase in pressure (similar to Prodanovic's work). Bubble sizes are smaller at higher pressures, and bubbles collapse faster at higher subcooling temperatures.

Next, the effect of the liquid mass flux on the bubble lift-off diameter was investigated under various conditions. The results show that the bubble lift-off diameter decreases with increasing mass flux. This is shown in Fig. 4. The effect of the liquid mass flux on the bubble sizes and lifetimes was more evident at lower heat fluxes. Since in this study, the available heat flux was relatively low, the effect of the mass flux was more pronounced. It was also observed that the bubble population increased with decreasing mass flux, particularly at low heat fluxes where the single-phase forced convection played an important role. 


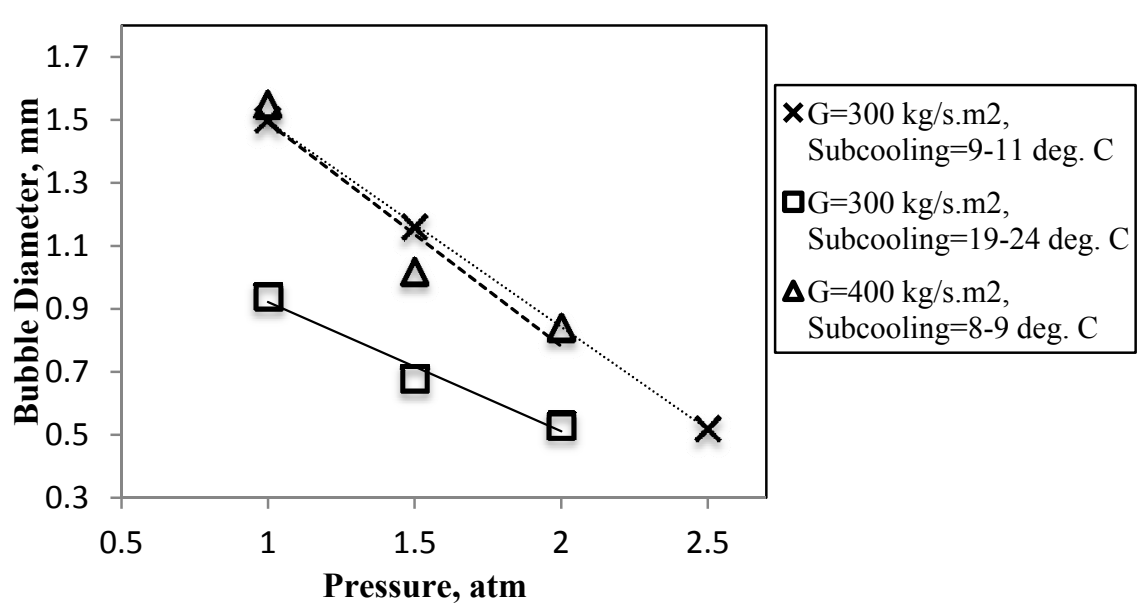

Figure 3: Effect of the liquid pressure on the bubble lift-off diameter (heat flux: $\left.124 \mathrm{~kW} / \mathrm{m}^{2}\right)$.

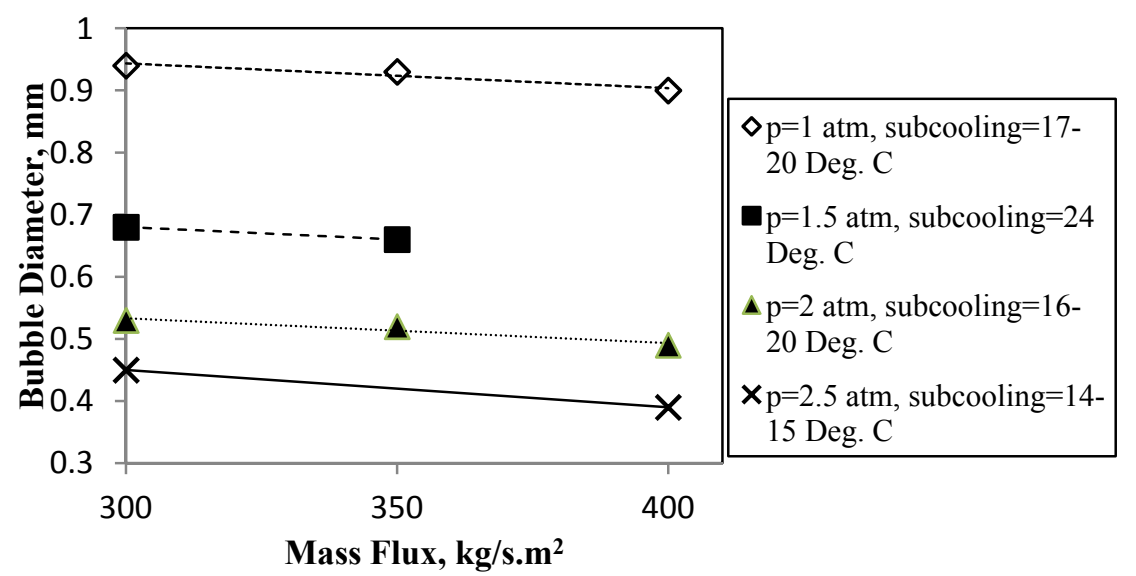

Figure 4: Effect of the mass flux on the bubble lift-off diameter (heat flux: $\left.124 \mathrm{~kW} / \mathrm{m}^{2}\right)$.

Fig. 5 shows the effect of liquid subcooling on bubble lift-off diameter. Bubble lift-off diameter decreases with increasing subcooling and mass flux. Our results are in agreement with those of Okawa et al. [9] who reported that bubbles slid longer distances under lower subcooling temperatures and higher mass fluxes. The results also showed that there was substantial variation in bubble liftoff diameter. This was the case even for the neighbouring sites considering that the heat flux was almost the same for all such nucleation sites, and even for cases in which the liquid subcooling between the inlet and outlet was $3-4^{\circ} \mathrm{C}$. This 
implies that although most models and correlations assume that the bubble liftoff diameter and nucleation frequency are only a function of external mass flow and heat flux, bubble characteristics, such as bubble diameter and frequency, depend on the microstructure of the nucleation cavities. Therefore, an adequate number of nucleation sites must be considered to obtain reliable results.

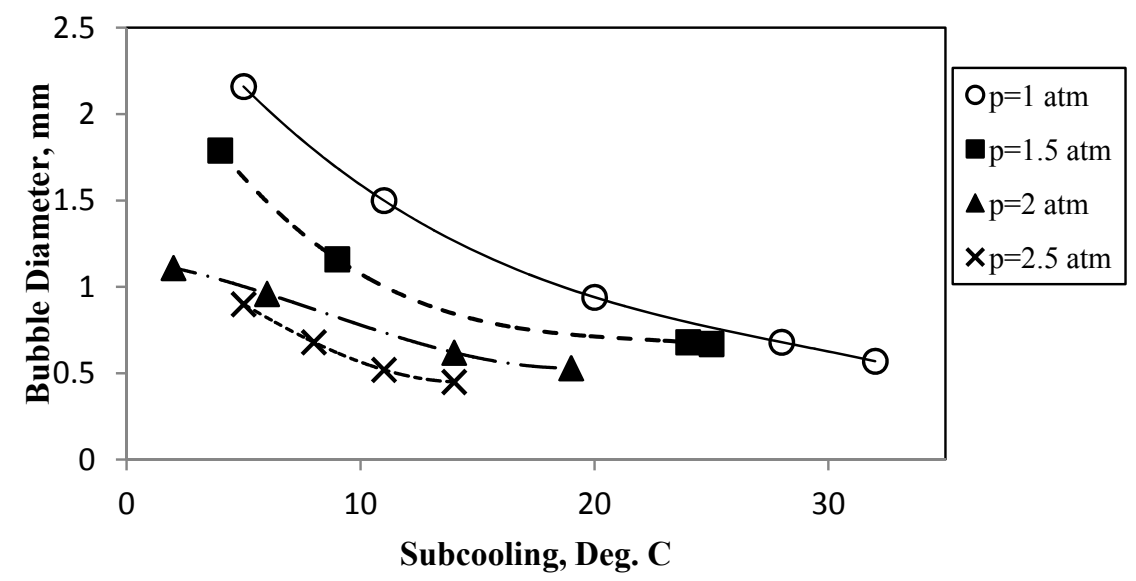

Figure 5: Effect of the fluid subcooling on the bubble lift-off diameter (heat flux: $130 \mathrm{~kW} / \mathrm{m}^{2}$, mass flux: $300 \mathrm{~kg} / \mathrm{m}^{2} . \mathrm{s}$ ).

\subsection{A correlation for bubble lift-off diameter}

In this study, a force balance at the point of lift-off in $y$-direction (normal direction to the flow) similar to Klausner et al. [1] was performed and simplifications have been applied to it as Zeng et al. [6] has suggested. Subsequently, the following model for the bubble lift-off diameter is obtained:

$$
D_{\text {lift }-o f f}=2 \sqrt[3]{\left(\frac{11 b^{4}}{\pi^{2}}\right)\left(\frac{\rho_{l}^{2} \alpha_{l} J a_{e}^{4}}{g\left(\rho_{l}-\rho_{g}\right)}\right)}
$$

where $J a_{e}$ is the effective Jakob number, $\alpha_{l}$ is the liquid thermal diffusivity, $\rho_{l}$ and $\rho_{g}$ are the liquid and gas densities, respectively.

This model strongly depends on the Jakob number. Since in the current study the surface temperature of the heater was not accurately measured, in order to calculate the Jakob number, the suggested correlation by Lui and Winterton [10] for calculating the wall superheat was used. Then, a model for the growth rate constant, $b$, was obtained based on the experimental data. These two correlations were then used and the predicted bubble lift-off diameters were calculated. The suggested model for $b$ is: 


$$
b-b_{0}=\sqrt{\frac{2}{3}} p^{0.3096}, \text { where } b_{0}=-0.304
$$

Calculated bubble lift-off diameters were compared with results obtained experimentally. The predictions were in good agreement with the experimentally determined values and the general trend of the predicted and experimental data were similar. With an increase in the subcooling margin, the bubble lift-off diameter decreased for a constant pressure. This was likely due to the fact that at higher subcooling, the effective wall superheat decreased due to the higher forced convection effect. In addition, both experimental and predicted trends showed that increasing pressure decreased the bubble lift-off diameter for the same subcooling.

In the present study, a database for the bubble lift-off diameter was built by integrating the data of the present work and the predictive capabilities of the proposed model. The results of the model were evaluated against the database. As shown in Fig. 6, the presented model provides reasonable agreement with our experimental work (average error of 20.78\%). The absolute error for each condition and the average error for all the conditions are defined as:

$$
\begin{gathered}
\text { Error }_{a b s}=\frac{\left|D_{l o, p r e d}-D_{l o, \exp }\right|}{D_{l o, \exp }} \times 100 \\
\text { Error }_{\text {avg }}=\frac{\sum_{i=1}^{i=N}\left(\frac{\left|D_{l o, p r e d}-D_{l o, \text { exp }}\right|}{D_{l o, \exp }}\right)}{N} \times 100 \%
\end{gathered}
$$

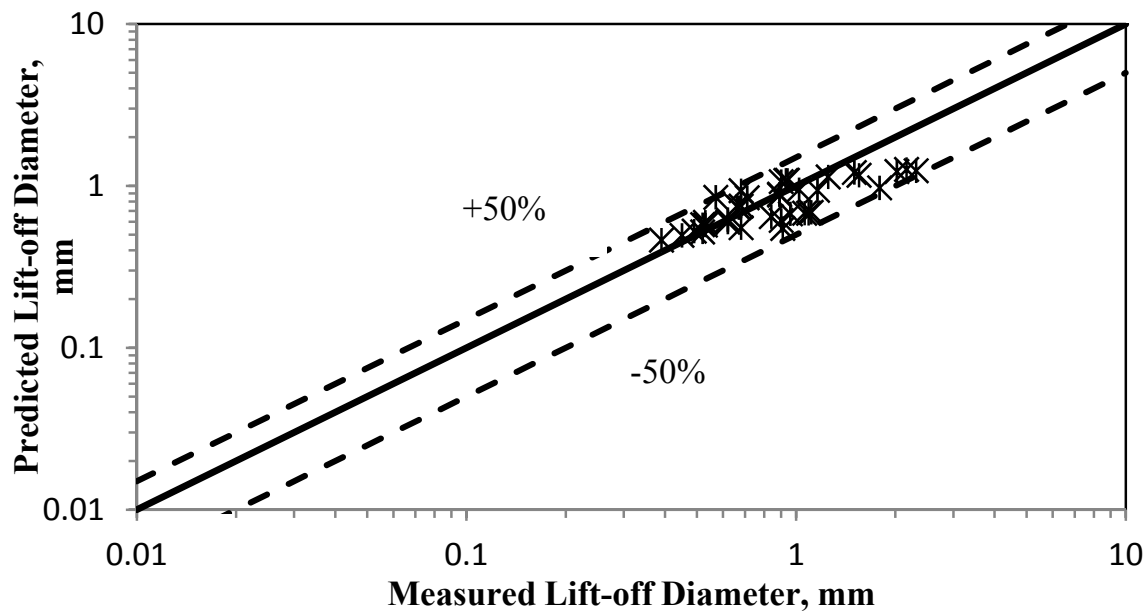

Figure 6: Prediction results of presented model against the present lift-off diameter data. 
There were several sources of error in our experiments, such as the error associated with the measurement methods, apparatus, and image processing. In addition, the model did not consider all the physical processes. For instance, shear forces are neglected and the contact angle at the time of lift-off is assumed to be zero. Although the relative velocities of each bubble in the direction of the flow were measured, the standard deviation was high. Therefore, the shear liftcoefficient could not be accurately determined. In addition, the definition of the relative bubble velocity with respect to the bubble centroid may have caused errors when bubbles deformed at lift-off. In the current model, $b$ as a function of pressure was used to provide the lowest average error of $20.78 \%$.

The current model was also compared with other commonly used models, as shown in fig. 7. The current model can be considered to be one of the best models for the prediction of the bubble lift-off diameter. Five models in addition to the current model are shown in this figure. The average errors associated with the Prodanovic et al. [3] model, Unal model [5], the Zeng et al. case [6], Situ et al. model [2], and Basu et al. model [4] are 27.8\%, 44.1\%, 78.2\%, 53.6\%, and 48.9\%, respectively.

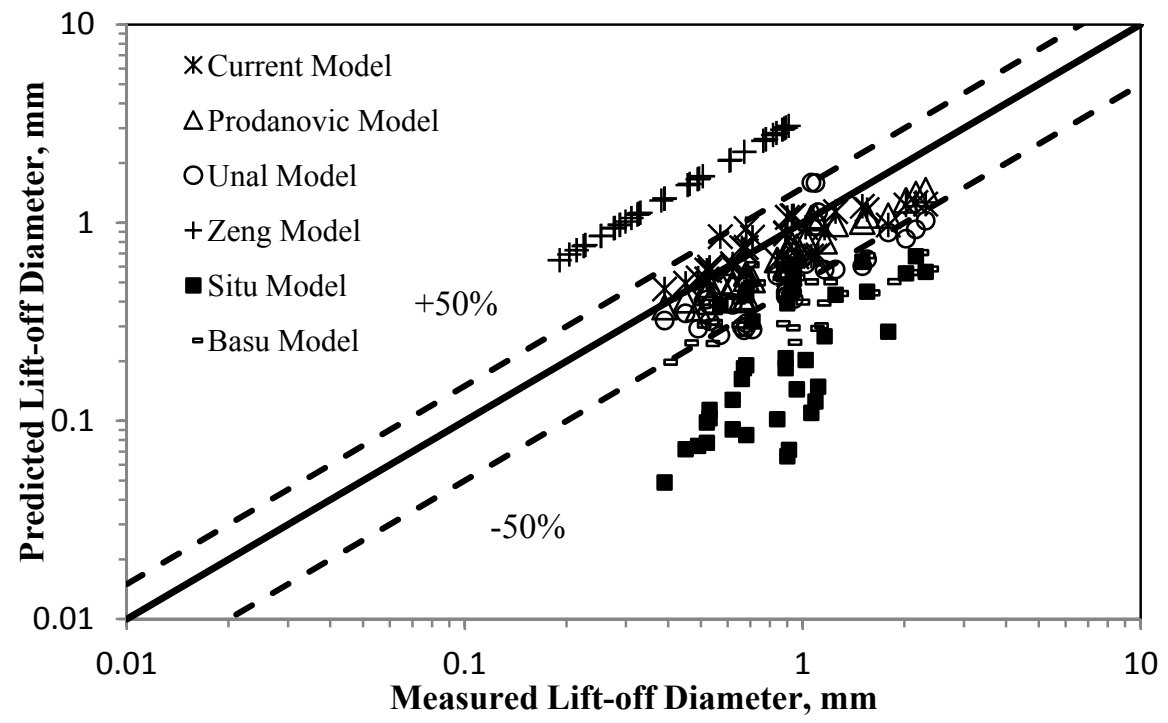

Figure 7: Prediction results of five models from literature alongside the current model's prediction results.

For the Zeng's case, we have used our model with the constant value for $b$ (i.e. $b=1.7$ ) as he suggested. This shows the highest average error and generally overpredicts the experimental result. This is mainly due to the use of a constant $b$ value. The second highest error is that of the Situ model. This model was developed for vertical chambers and the main reason for its error is due to their assumption that the bubble sliding velocity is half the local liquid velocity. This assumption does not coincide with other researchers' observations. Basu and 
Unal's models also show high errors which is due to the fact that their models were developed for vertical and not horizontal flows. In addition, Unal's model predicts the maximum bubble diameter and not the lift-off diameter. The best result was seen in Prodanovic's model with only $27.8 \%$ of error which was still almost $8 \%$ higher than the current model's error. These comparisons clearly prove that vertical models should not be used for horizontal flows and that for better results more work must be performed for horizontal flow conditions.

\section{Conclusion}

An experimental study was performed on bubble nucleation on a heated zirconium rod horizontally located in a subcooled flow channel for 37 test conditions. The project investigated two-phase subcooled flow boiling inside a rectangular horizontal channel with heat supplied by an electrically heated rod while vapour bubbles were created on a horizontal wall in a uniform flow. The purpose of this investigation was to find a preliminary empirical relationship for bubble lift-off diameter and growth rate constant under different experimental conditions. These experiments were designed in order to validate the predictions, and to extend the predictive capacity to conditions that actually occur in practice. A high-speed camera was used for filming bubble growth and detachment from its side view. MatLAB image processing code was developed for data acquisition of images acquired during experiments. Experiments were performed for inlet water subcooling of $32-1{ }^{\circ} \mathrm{C}$, pressures of $1-2.5 \mathrm{~atm}$, and flow rates of $300-400 \mathrm{~kg} / \mathrm{m}^{2} \mathrm{~s}$ at a constant heat flux of $124 \mathrm{~kW} / \mathrm{m}^{2}$.

Bubble growth rate and maximum bubble radii obtained in this study were obtained using Zuber's bubble growth model. Lift-off bubble radius was correlated with wall superheat $(J a)$, pressure, and indirectly to liquid bulk subcooling (Zeng et al. [6]). The bubble growth rate constant, $b$, is assumed to be $b=1.73$ in most applications. The present study showed that the growth rate constant cannot be assumed to be constant and is in fact a strong function of flow pressure. Although more experiments should be performed at higher pressures to validate this model, in using this new model, the prediction error of the bubble lift-off diameter was significantly reduced. Therefore, a new empirical correlation for $b$ as a function of experimental pressure was found which better predicted the experimental data.

\section{References}

[1] Klausner, J.F., Mei, R., Bernhard, D.M., Zeng, L.Z., "Vapor bubble departure in forced convection boiling," Int. J. Heat Mass Transfer, vol. 36, no. 3, pp. 651-662, 1993.

[2] Situ, R., Hibiki, T., Ishii, M., Mori, M., "Bubble lift-off size in forced convective subcooled boiling flow," Int. J. Heat Mass Transfer, vol. 48, pp. 5536-3348, 2005. 
[3] Prodanovic, V., Fraser, D., Salcudean, M.,, "Bubble behavior in subcooled flow boiling of water at low pressures and low flow rates," Int. J. Multiphase Flow, vol. 28, pp. 1-19, 2002.

[4] Basu, N., Warrier, G.R., Dhir, V.K., "Onset of nucleate boiling and active nucleation site density during subcooled flow boiling," J. Heat Transfer, vol. 124, pp. 717-728, 2002.

[5] H. Unal, "Maximum bubble diameter, maximum bubble growth time and bubble growth rate during the subcooled nucleate flow boiling of water up to 17.7MPa," Int. J. Heat Mass Transfer, vol. 19, pp. 643-649, 1976.

[6] L. Z. Zeng, J. F. Klausner, D. M. Bernhard and R. Mei, "A unified model for the prediction of bubble detachment diameters in boiling systems," Int. J. Heat Mass Transfer, vol. II, no. 36, pp. 2271-2279, 1993.

[7] N. Zuber, "The dynamics of vapor bubbles in non-uniform temperature fields," Int. J. Heat Mass Transfer, no. 2, pp. 83-98, 1961.

[8] V. I. Tolubinsky and D. M. Kostanchuk, "Vapour bubbles growth rate and heat transfer intensity at subcooled water boiling," in 4th International Heat Transfer Conference, 1970.

[9] T. Okawa, T. Ishida, I. Kataoka and M. Mori, "Bubble rise characteristics after the departure from a nucleation site in vertical upflow boiling of subcooled water," Nucl. Eng. Des., no. 235, pp. 1149-1161, 2005.

[10] Z. Liu and R. H. S. Winterton, "A General Correlation for Saturated and Subcooled Flow Boiling in Tubes and Annuli, Based on a Nucleate Pool Boiling Equation," Int. J. Heat Mass Transter, no. 34, pp. 2759-2766, 1991. 